Research Article

\title{
Convergence of Implicit and Explicit Schemes for an Asymptotically Nonexpansive Mapping in $q$-Uniformly Smooth and Strictly Convex Banach Spaces
}

\author{
Meng Wen, Changsong Hu, and Zhiyu Wu \\ Department of Mathematics, Hubei Normal University, Hubei, Huangshi 435002, China \\ Correspondence should be addressed to Changsong Hu, huchang1004@yahoo.com.cn \\ Received 24 April 2012; Accepted 23 June 2012 \\ Academic Editor: Hong-Kun Xu \\ Copyright (c) 2012 Meng Wen et al. This is an open access article distributed under the Creative \\ Commons Attribution License, which permits unrestricted use, distribution, and reproduction in \\ any medium, provided the original work is properly cited. \\ We introduce a new iterative scheme with Meir-Keeler contractions for an asymptotically \\ nonexpansive mapping in $q$-uniformly smooth and strictly convex Banach spaces. We also proved \\ the strong convergence theorems of implicit and explicit schemes. The results obtained in this \\ paper extend and improve many recent ones announced by many others.
}

\section{Introduction}

Let $E$ be a real Banach space. With $J: E \rightarrow 2^{E^{*}}$, we denote the normalized duality mapping given by

$$
J(x)=\left\{f \in E^{*}:\langle x, f\rangle=\|x\|^{2},\|f\|=\|x\|\right\},
$$

where $\langle\cdot, \cdot\rangle$ denotes the generalized duality pairing and $E^{*}$ the dual space of $E$. In the sequel we will donate single-valued duality mappings by $j$. Given $q>1$, by $J_{q}$ we will denote the generalized duality mapping given by

$$
J_{q}(x)=\left\{f \in E^{*}:\langle x, f\rangle=\|x\|^{q},\|f\|=\|x\|^{q-1}\right\} .
$$


We recall that the following relation holds:

$$
J_{q}(x)=\|x\|^{q-2} J(x)
$$

for $x \neq 0$. defined by

We recall that the modulus of smoothness of $E$ is the function $\rho_{E}:[0, \infty) \rightarrow[0, \infty)$

$$
\rho_{E}(t):=\sup \left\{\frac{1}{2}(\|x+y\|+\|x-y\|)-1:\|x\| \leq 1,\|y\| \leq t\right\}
$$

$E$ is said to be uniformly smooth if $\lim _{t \rightarrow 0}\left(\rho_{E}(t) / t\right)=0$.

Let $q>1$. $E$ is said to be $q$-uniformly smooth if there exists a constant $c>0$ such that $\rho_{E}(t) \leq c t^{q}$. Examples of such spaces are Hilbert spaces and $L_{p}\left(\right.$ or $\left.l_{p}\right)$.

We note that a $q$-uniformly smooth Banach space is uniformly smooth. This implies that its norm uniformly Fréchet differentiable (see [1]).

If $E$ is uniformly smooth, then the normalized duality map $j$ is single-valued and norm to norm uniformly continuous.

Let $E$ be a real Banach space and $C$ is a nonempty closed convex subset of $E$. A mapping $T: C \rightarrow C$ is said to be asymptotically nonexpansive if there exists a sequence $\left\{h_{n}\right\} \subset[0, \infty)$ with $\lim _{n \rightarrow \infty} h_{n}=0$ such that

$$
\left\|T^{n} x-T^{n} y\right\| \leq\left(1+h_{n}\right)\|x-y\|, \quad x, y \in C, n \geq 1,
$$

and $F(T)$ denotes the set of fixed points of the mapping $T$; that is, $F(T)=\{x \in C: T x=x\}$. For asymptotically nonexpansive self-map $T$, it is well known that $F(T)$ is closed and convex (see e.g., [2]).

Theorem 1.1 (Banach [3]). Let $(X, d)$ be a complete metric space and let $f$ be a contraction on $X$; that is, there exists $r \in(0,1)$ such that $d(f(x), f(y)) \leq r d(x, y)$ for all $x, y \in X$. Then $f$ has a unique fixed point.

Theorem 1.2 (Meir and Keeler [4]). Let $(X, d)$ be a complete metric space and let $\phi$ be a MeirKeeler contraction $(M K C)$ on $X$, that is, for every $\varepsilon>0$, there exists $\delta>0$ such that $d(x, y)<\varepsilon+\delta$ implies $d(\phi(x), \phi(y))<\varepsilon$ for all $x, y \in X$. Then $\phi$ has a unique fixed point.

This theorem is one of generalizations of Theorem 1.1, because contractions are MeirKeeler contractions.

We recall that, given a $q$-uniformly smooth and strictly convex Banach space $E$ with a generalized duality map $J_{q}: E \rightarrow E^{*}$ and $C$ a subset of $E$, a mapping $F: C \rightarrow C$ is called

(1) $k^{\prime}$-Lipschitzian, if there exists a constant $k^{\prime}>0$ such that

$$
\|F x-F y\| \leq k^{\prime}\|x-y\|
$$

holds for every $x$ and $y \in C$; 
(2) $\eta$-strongly monotone, if there exists a constant $\eta>0$ such that

$$
\left\langle F x-F y, j_{q}(x-y)\right\rangle \geq \eta\|x-y\|^{q}
$$

holds for every $x, y \in C$ and $j_{q}(x-y) \in J_{q}(x-y)$.

In 2010, Ali and Ugwunnadi [5] introduced and considered the following iterative scheme:

$$
\begin{gathered}
x_{0} \in H, \\
x_{n+1}=\beta_{n} x_{n}+\left(1-\beta_{n}\right) y_{n} \\
y_{n}=\left(I-\alpha_{n} A\right) T_{i(n+1)}^{p(n+1)} x_{n}+\alpha_{n} \gamma f\left(x_{n}\right), \quad \forall n \geq 1,
\end{gathered}
$$

where $T_{1}, T_{2}, \ldots, T_{N}$ a family of asymptotically nonexpansive self-mappings of $H$ with sequences $\left\{1+k_{p(n)}^{i(n)}\right\}$, such that $k_{p(n)}^{i(n)} \rightarrow 0$ as $n \rightarrow \infty$ and $f: H \rightarrow H$ are a contraction mapping with coefficient $\alpha \in(0,1)$. Let $A$ be a strongly positive-bounded linear operator with coefficient $\bar{\gamma}>0$, and $0<\gamma<\bar{\gamma} / \alpha$. They proved the strong convergence of the implicit and explicit schemes for a common fixed point of the family $T_{1}, T_{2}, \ldots, T_{N}$, which solves the variational inequality $\langle(A-\gamma f) \bar{x}, \bar{x}-x\rangle \leq 0$, for all $x \in \bigcap_{i=1}^{N} \operatorname{Fix}\left(T_{i}\right)$.

Motivated and inspired by the results of Ali and Ugwunnadi [5], we introduced an iterative scheme as follows. for $x_{1}=x \in C$,

$$
\begin{gathered}
x_{n+1}=\beta_{n} x_{n}+\left(1-\beta_{n}\right) y_{n}, \\
y_{n}=\left(I-\mu \alpha_{n} F\right) T^{n} x_{n}+\alpha_{n} \gamma \phi\left(x_{n}\right), \quad \forall n \geq 1,
\end{gathered}
$$

where $T$ is an asymptotically nonexpansive self-mapping of $C$ with sequences $\left\{1+h^{n}\right\}$, such that $h^{n} \rightarrow 0$ as $n \rightarrow \infty$ and $\phi: C \rightarrow C$ are a Meir-Keeler contraction (MKC, forshort). Let $F$ is a $k^{\prime}$-Lipschitzian and $\eta$-strongly monotone operator with $0<\mu<\min \left\{\left(q \eta / C_{q}\left(k^{\prime}\right)^{q}\right)^{1 /(q-1)}, 1\right\}$. We will prove the strong convergence of the implicit and explicit schemes for a fixed point of $T$, which solves the variational inequality $\left\langle(\gamma \phi-\mu F) p, J_{q}(z-p)\right\rangle \leq 0$, for $z \in F(T)$. Our results improve and extend the results of Ali and Ugwunnadi [5] for an asymptotically nonexpansive mapping in the following aspects:

(i) Hilbert space is replaced by a $q$-uniformly smooth and strictly convex Banach space;

(ii) contractive mapping is replaced by a MKC;

(iii) Theorems 3.1 and 4.1 extend the results of Ali and Ugwunnadi [5] from a strongly positive-bounded linear operator $A$ to a $k^{\prime}$-Lipschitzian and $\eta$-strongly monotone operator $F$.

\section{Preliminaries}

In order to prove our main results, we need the following lemmas. 
Lemma 2.1 (see [6]). Let $q>1$ and E be a q-uniformly smooth Banach space, then there exists a constant $C_{q}>0$ such that

$$
\|x+y\|^{q} \leq\|x\|^{q}+q\left\langle y, j_{q}(x)\right\rangle+C_{q}\|y\|^{q}, \quad \forall x, y \in E .
$$

Lemma 2.2 (see [7, Lemma 2.3]). Let $\phi$ be a MKC on a convex subset $C$ of a Banach space E. Then for each $\varepsilon>0$, there exists $r \in(0,1)$ such that

$$
\|x-y\| \geq \varepsilon \text { implies }\|\phi x-\phi y\| \leq r\|x-y\| \quad \forall x, y \in C .
$$

Lemma 2.3 (see [8]). Let $\left\{x_{n}\right\}$ and $\left\{z_{n}\right\}$ be bounded sequences in a Banach space $E$ and $\left\{\gamma_{n}\right\}$ be a sequence in $[0,1]$ which satisfies the following condition:

$$
0<\liminf _{n \rightarrow \infty} \gamma_{n} \leq \limsup _{n \rightarrow \infty} \gamma_{n}<1
$$

Suppose that $x_{n+1}=\gamma_{n} x_{n}+\left(1-\gamma_{n}\right) z_{n}, n \geq 0$, and $\lim \sup _{n \rightarrow \infty}\left(\left\|z_{n+1}-z_{n}\right\|-\left\|x_{n+1}-x_{n}\right\|\right) \leq 0$. Then $\lim _{n \rightarrow \infty}\left\|z_{n}-x_{n}\right\|=0$.

Lemma 2.4 (see $[9,10])$. Let $\left\{s_{n}\right\}$ be a sequence of nonnegative real numbers satisfying

$$
s_{n+1} \leq\left(1-\lambda_{n}\right) s_{n}+\lambda_{n} \delta_{n}+\gamma_{n}, \quad n \geq 0,
$$

where $\left\{\lambda_{n}\right\},\left\{\delta_{n}\right\}$ and $\left\{\gamma_{n}\right\}$ satisfy the following conditions: (i) $\left\{\lambda_{n}\right\} \subset[0,1]$ and $\sum_{n=0}^{\infty} \lambda_{n}=\infty$, (ii) $\lim \sup _{n \rightarrow \infty} \delta_{n} \leq 0$ or $\sum_{n=0}^{\infty} \lambda_{n} \delta_{n}<\infty$, (iii) $\gamma_{n} \geq 0(n \geq 0), \sum_{n=0}^{\infty} \gamma_{n}<\infty$. Then $\lim _{n \rightarrow \infty} s_{n}=0$.

Lemma 2.5 (see [11]). Let $C$ be a nonempty closed convex subset of a uniformly convex Banach space $E$ and $T: C \rightarrow E$ is an asymptotically nonexpansive mapping with $F(T) \neq \emptyset$. Then the mapping $I-T$ is demiclosed at zero, that is, $x_{n} \rightarrow x$ and $\left\|x_{n}-T x_{n}\right\| \rightarrow 0$, then $x=T x$.

Lemma 2.6. Let $F$ be a $k^{\prime}$-Lipschitzian and $\eta$-strongly monotone operator on a q-uniformly smooth Banach space $E$ with $k^{\prime}>0, \eta>0,0<t<1$ and $0<\mu<\min \left\{\left(q \eta / C_{q}\left(k^{\prime}\right)^{q}\right)^{1 /(q-1)}, 1\right\}$. Then $S=$ $(I-t \mu F): E \rightarrow E$ is a contraction with contractive coefficient $1-t \tau$ and $\tau=\left(q \mu \eta-C_{q} \mu^{q}\left(k^{\prime}\right)^{q}\right) / q$.

Proof. From (2.1), we have

$$
\begin{aligned}
\|S x-S y\|^{q} & =\|x-y-t \mu(F x-F y)\|^{q} \\
& \leq\|x-y\|^{q}+q\left\langle-t \mu(F x-F y), J_{q}(x-y)\right\rangle+C_{q}\|-t \mu(F x-F y)\|^{q} \\
& \leq\|x-y\|^{q}-t q \mu \eta\|x-y\|^{q}+t C_{q} \mu^{q}\left(k^{\prime}\right)^{q}\|x-y\|^{q} \\
& =\left[1-t\left(q \mu \eta-C_{q} \mu^{q}\left(k^{\prime}\right)^{q}\right)\right]\|x-y\|^{q} \\
& \leq\left[1-t \frac{q \mu \eta-C_{q} \mu^{q}\left(k^{\prime}\right)^{q}}{q}\right]^{q}\|x-y\|^{q} \\
& =(1-t \tau)^{q}\|x-y\|^{q}
\end{aligned}
$$


where $\tau=\left(q \mu \eta-C_{q} \mu^{q}\left(k^{\prime}\right)^{q}\right) / q$, and

$$
\|S x-S y\| \leq(1-t \tau)\|x-y\|
$$

Hence $S$ is a contraction with contractive coefficient $1-t \tau$.

Lemma 2.7 (see [5, Lemma 2.9]). Let $T: E \rightarrow$ E be a uniformly Lipschitzian with a Lipschitzian constant $L \geq 1$, that is, there exists a constant $L \geq 1$ such that

$$
\left\|T^{n} x-T^{n} y\right\| \leq L\|x-y\|, \quad \forall x, y \in E .
$$

Lemma 2.8 (see, e.g., Mitrinović [12, page 63]). Let $q>1$. Then the following inequality holds:

$$
a b \leq \frac{1}{q} a^{q}+\frac{q-1}{q} b^{q /(q-1)},
$$

for arbitrary positive real numbers $a, b$.

\section{Main Result}

Theorem 3.1. Let $E$ be a q-uniformly smooth and strictly convex Banach space, and $C$ a nonempty closed convex subset of $E$ such that $C \pm C \subset C$ and have a weakly sequentially continuous duality mapping $J_{q}$ from $E$ to $E^{*}$. Let $T: C \rightarrow C$ be an asymptotically nonexpansive mapping with sequences $\left\{1+h_{n}\right\}$, such that $h_{n} \rightarrow 0$ as $n \rightarrow \infty$ and $F^{*}:=F(T) \neq \emptyset$. Let $D$ be a bounded subset of $C$ such that $\sup _{x \in D}\left\|T^{n+1} x-T^{n} x\right\| \rightarrow 0$. Let $F$ be a $k^{\prime}$-Lipschitzian and $\eta$-strongly monotone operator on $C$ with $0<\mu<\min \left\{\left(q \eta / C_{q}\left(k^{\prime}\right)^{q}\right)^{1 /(q-1)}, 1\right\}$, and $\phi$ be a MKC on $C$ with $0<\gamma<\left(q \mu \eta-C_{q} \mu^{q}\left(k^{\prime}\right)^{q}\right) / q=\tau$. Let $\left\{\alpha_{n}\right\}$ be a sequence in $(0,1)$ satisfying the following conditions:

(A1) $\lim _{n \rightarrow \infty} \alpha_{n}=0$;

(A2) $\lim _{n \rightarrow \infty}\left(h_{n} / \alpha_{n}\right)=0$.

Let $\left\{x_{n}\right\}$ be defined by

$$
x_{n}=\alpha_{n} \gamma \phi\left(x_{n}\right)+\left(I-\alpha_{n} \mu F\right) T^{n} x_{n} .
$$

Then, $\left\{x_{n}\right\}$ converges to a fixed point say $p$ in $F^{*}$ which solves the variational inequality

$$
\left\langle(\mu F-\gamma \phi) p, J_{q}(p-z)\right\rangle \leq 0, \quad \forall z \in F^{*} .
$$


Proof. Let $p \in F^{*}$. Since $\alpha_{n} \rightarrow 0$ and $h_{n} / \alpha_{n} \rightarrow 0$ as $n \rightarrow \infty$, then $\left(1-\alpha_{n} \tau\right)\left(h_{n} / \alpha_{n}\right) \rightarrow 0$ as $n \rightarrow \infty$, so $\exists N_{0} \in N$ such that for all $n \geq N_{0}, \alpha_{n}<\left(k^{\prime}\right)^{-1}$ and $\left(1-\alpha_{n} \tau\right)\left(h_{n} / \alpha_{n}\right)<(1 / 2)(\tau-\gamma)$. Thus, for $n \geq N_{0}$

$$
\begin{aligned}
\left\|x_{n}-p\right\|^{q}= & \left\langle\alpha_{n} \gamma \phi\left(x_{n}\right)+\left(I-\alpha_{n} \mu F\right) T^{n} x_{n}-p, J_{q}\left(x_{n}-p\right)\right\rangle \\
= & \alpha_{n}\left\langle\gamma \phi\left(x_{n}\right)-\mu F p, J_{q}\left(x_{n}-p\right)\right\rangle+\left\langle\left(I-\alpha_{n} \mu F\right) T^{n} x_{n}-\left(I-\alpha_{n} \mu F\right) p, J_{q}\left(x_{n}-p\right)\right\rangle \\
= & \alpha_{n}\left\langle\gamma \phi\left(x_{n}\right)-\gamma \phi(p), J_{q}\left(x_{n}-p\right)\right\rangle+\alpha_{n}\left\langle\gamma \phi(p)-\mu F p, J_{q}\left(x_{n}-p\right)\right\rangle \\
& +\left\langle\left(I-\alpha_{n} \mu F\right) T^{n} x_{n}-\left(I-\alpha_{n} \mu F\right) p, J_{q}\left(x_{n}-p\right)\right\rangle \\
\leq & \alpha_{n} \gamma\left\|x_{n}-p\right\|^{q}+\left(1-\alpha_{n} \tau\right)\left(1+h_{n}\right)\left\|x_{n}-p\right\|^{q}+\alpha_{n}\left\langle\gamma \phi(p)-\mu F p, J_{q}\left(x_{n}-p\right)\right\rangle \\
= & {\left[1-\alpha_{n}(\tau-\gamma)+\left(1-\alpha_{n} \tau\right) h_{n}\right]\left\|x_{n}-p\right\|^{q}+\alpha_{n}\left\langle\gamma \phi(p)-\mu F p, J_{q}\left(x_{n}-p\right)\right\rangle } \\
\leq & \frac{\left\langle\gamma \phi(p)-\mu F p, J_{q}\left(x_{n}-p\right)\right\rangle}{(\tau-\gamma)-\left(1-\alpha_{n} \tau\right)\left(h_{n} / \alpha_{n}\right)} \\
\leq & \frac{\left\langle\gamma \phi(p)-\mu F p, J_{q}\left(x_{n}-p\right)\right\rangle}{(\tau-\gamma)-(1 / 2)(\tau-\gamma)} \\
\leq & \frac{2\|\gamma \phi(p)-\mu F p\|\left\|x_{n}-p\right\|^{q-1}}{\tau-\gamma} .
\end{aligned}
$$

Therefore,

$$
\left\|x_{n}-p\right\| \leq \frac{2\|\gamma \phi(p)-\mu F p\|}{\tau-\gamma}
$$

Thus, $\left\{x_{n}\right\}$ is bounded and therefore $\left\{\phi\left(x_{n}\right)\right\}$ and $\left\{\mu F T^{n} x_{n}\right\}$ are also bounded. Also from (3.1), we have

$$
\left\|x_{n}-T^{n} x_{n}\right\|=\alpha_{n}\left\|\gamma \phi\left(x_{n}\right)-\mu F T^{n} x_{n}\right\| \longrightarrow 0 \text { as } n \longrightarrow \infty
$$

From (3.5) and $\left\|T^{n+1} x_{n}-T^{n} x_{n}\right\| \rightarrow 0$, we obtain

$$
\begin{gathered}
\left\|T^{n+1} x_{n}-x_{n}\right\| \leq\left\|T^{n+1} x_{n}-T^{n} x_{n}\right\|+\left\|T^{n} x_{n}-x_{n}\right\| \longrightarrow 0, \\
\left\|T^{n+1} x_{n}-T x_{n}\right\| \leq\left(1+h_{1}\right)\left\|T^{n} x_{n}-x_{n}\right\| \longrightarrow 0,
\end{gathered}
$$

Thus,

$$
\left\|T x_{n}-x_{n}\right\| \leq\left\|T x_{n}-T^{n+1} x_{n}\right\|+\left\|T^{n+1} x_{n}-x_{n}\right\| \longrightarrow 0 .
$$


Since $\left\{x_{n}\right\}$ is bounded, now assume that $p$ is a weak limit point of $\left\{x_{n}\right\}$ and a subsequence $\left\{x_{n_{j}}\right\}$ of $\left\{x_{n}\right\}$ converges weakly to $p$. Then, by Lemma 2.5 and (3.7), we have that $p$ is a fixed point of $T$, hence $p \in F^{*}$.

Next we observe that the solution of the variational inequality (3.2) in $F^{*}$ is unique. Assume that $\tilde{q}, p \in F^{*}$ are solutions of the inequality (3.2), without loss of generality, we may assume that there is a number $\varepsilon$ such that $\|p-\tilde{q}\| \geq \varepsilon$. Then by Lemma 2.2, there is a number $r$ such that $\|\phi p-\phi \tilde{q}\| \leq r\|p-\tilde{q}\|$. From (3.2), we know

$$
\begin{aligned}
& \left\langle(\mu F-\gamma \phi) p, J_{q}(p-\tilde{q})\right\rangle \leq 0 \\
& \left\langle(\mu F-\gamma \phi) \tilde{q}, J_{q}(\tilde{q}-p)\right\rangle \leq 0 .
\end{aligned}
$$

Adding (3.8) and (3.9), we have

$$
\left\langle(\mu F-\gamma \phi) p-(\mu F-\gamma \phi) \tilde{q}, J_{q}(p-\tilde{q})\right\rangle \leq 0
$$

Noticing that

$$
\begin{aligned}
\left\langle(\mu F-\gamma \phi) p-(\mu F-\gamma \phi) \tilde{q}, J_{q}(p-\tilde{q})\right\rangle & =\left\langle\mu F p-\mu F \tilde{q}, J_{q}(p-\tilde{q})\right\rangle-\left\langle\gamma \phi p-\gamma \phi \tilde{q}, J_{q}(p-\tilde{q})\right\rangle \\
& \geq \mu \eta\|p-\tilde{q}\|^{q}-\gamma\|\phi p-\phi \tilde{q}\|\|p-\tilde{q}\|^{q-1} \\
& \geq \mu \eta\|p-\tilde{q}\|^{q}-\gamma r\|p-\tilde{q}\|^{q} \\
& \geq(\mu \eta-\gamma r)\|p-\tilde{q}\|^{q} \\
& \geq(\mu \eta-\gamma r) \varepsilon^{q} \\
& >0 .
\end{aligned}
$$

Therefore $p=\tilde{q}$. That is, $p \in F^{*}$ is the unique solution of (3.2).

Finally, we show that $x_{n} \rightarrow p$ as $n \rightarrow \infty$. From (3.3), we get

$$
\begin{aligned}
\left\|x_{n}-p\right\|^{q} & \leq \frac{\alpha_{n}\left\langle\gamma \phi(p)-\mu F p, J_{q}\left(x_{n}-p\right)\right\rangle}{\alpha_{n}(\tau-\gamma)-\left(1-\alpha_{n} \tau\right) h_{n}} \\
& =\frac{\left\langle\gamma \phi(p)-\mu F p, J_{q}\left(x_{n}-p\right)\right\rangle}{(\tau-\gamma)-\left(1-\alpha_{n} \tau\right)\left(h_{n} / \alpha_{n}\right)}
\end{aligned}
$$

and in particular

$$
\left\|x_{n_{j}}-p\right\|^{q} \leq \frac{\left\langle\gamma \phi(p)-\mu F p, J_{q}\left(x_{n_{j}}-p\right)\right\rangle}{(\tau-\gamma)-\left(1-\alpha_{n_{j}} \tau\right)\left(h_{n_{j}} / \alpha_{n_{j}}\right)} .
$$


Since $x_{n_{j}} \rightarrow p$, from the above inequality and $J_{q}$ is a weakly sequentially continuous duality mapping, we have $x_{n_{j}} \rightarrow p$ as $j \rightarrow \infty$. Next, we show that $p$ solves the variational inequality (3.2). Indeed, from the relation

$$
x_{n}=\alpha_{n} \gamma \phi\left(x_{n}\right)+\left(I-\alpha_{n} \mu F\right) T^{n} x_{n}
$$

we get

$$
(\mu F-\gamma \phi) x_{n}=-\frac{1}{\alpha_{n}}\left[\left(I-T^{n}\right) x_{n}-\alpha_{n} \mu F x_{n}+\alpha_{n} \mu F T^{n} x_{n}\right]
$$

So, for any $z \in F^{*}$

$$
\begin{aligned}
\langle(\mu F- & \left.\gamma \phi) x_{n}, J_{q}\left(x_{n}-z\right)\right\rangle \\
= & -\frac{1}{\alpha_{n}}\left\langle\left(I-T^{n}\right) x_{n}-\alpha_{n} \mu F x_{n}+\alpha_{n} \mu F T^{n} x_{n}, J_{q}\left(x_{n}-z\right)\right\rangle \\
= & -\frac{1}{\alpha_{n}}\left\langle\left(I-T^{n}\right) x_{n}-\left(I-T^{n}\right) z, J_{q}\left(x_{n}-z\right)\right\rangle \\
& +\left\langle\left(\mu F-\mu F T^{n}\right) x_{n}, J_{q}\left(x_{n}-z\right)\right\rangle \\
\leq & -\frac{1}{\alpha_{n}}\left\|x_{n}-z\right\|^{q}+\frac{1}{\alpha_{n}}\left(1+h_{n}\right)\left\|x_{n}-z\right\|^{q}+\left\langle\left(\mu F-\mu F T^{n}\right) x_{n}, J_{q}\left(x_{n}-z\right)\right\rangle \\
\leq & \frac{h_{n}}{\alpha_{n}}\left\|x_{n}-z\right\|^{q}+\left\langle\left(\mu F-\mu F T^{n}\right) x_{n}, J_{q}\left(x_{n}-z\right)\right\rangle .
\end{aligned}
$$

Now replacing $n$ in (3.16) with $n_{j}$ and letting $j \rightarrow \infty$, using $\left(\mu F-\mu F T^{n}\right) x_{n_{j}} \rightarrow\left(\mu F-\mu F T^{n}\right) p=$ 0 for $p \in F^{*}$, and the fact that $x_{n_{j}} \rightarrow p$ as $j \rightarrow \infty$, we obtain $\left\langle(\mu F-\gamma \phi) p, J_{q}(p-z)\right\rangle \leq 0, \forall z \in F^{*}$. This implies that $p \in F^{*}$ is a solution of the variational inequality (3.2). Every weak limit of $\left\{x_{n}\right\}$ say $p$ belongs to $F^{*}$. Furthermore, $p$ is a strong limit of $\left\{x_{n}\right\}$ that solves the variational inequality (3.2). As this solution is unique we get that $x_{n} \rightarrow p$ as $n \rightarrow \infty$. This completes the proof.

Corollary 3.2. Let $E$ be a q-uniformly smooth and strictly convex Banach space, and let $C$ be a nonempty closed convex subset of $E$ such that $C \pm C \subset C$ and have a weakly sequentially continuous duality mapping $J_{q}$ from $E$ to $E^{*}$. Let $T: C \rightarrow C$ be a nonexpansive mapping. Let $\left\{\alpha_{n}\right\}$ be a sequence in $(0,1)$ satisfying $\lim _{n \rightarrow \infty} \alpha_{n}=0$. Let $\phi$ and $F$ be as in Theorem 3.1. For $T$, let $\left\{x_{n}\right\}$ be defined by

$$
x_{n}=\alpha_{n} \gamma \phi\left(x_{n}\right)+\left(I-\alpha_{n} \mu F\right) T x_{n} .
$$

Then, $\left\{x_{n}\right\}$ converges to a fixed point say $p$ in $F^{*}$ which solves the variational inequality (3.2).

\section{Explicit Algorithm}

Theorem 4.1. Let $E$ be a q-uniformly smooth and strictly convex Banach space, and let $C$ be a nonempty closed convex subset of $E$ such that $C \pm C \subset C$ and have a weakly sequentially continuous 
duality mapping $J_{q}$ from $E$ to $E^{*}$. Let $T: C \rightarrow C$ be an asymptotically nonexpansive mapping with sequences $\left\{1+h_{n}\right\}$, such that $h_{n} \rightarrow 0$ as $n \rightarrow \infty$ and $F^{*}:=F(T) \neq \emptyset$. Let $D$ be a bounded subset of $C$ such that $\sup _{x \in D}\left\|T^{n+1} x-T^{n} x\right\| \rightarrow 0$. Let $F$ be a $k^{\prime}$-Lipschitzian and $\eta$-strongly monotone operator on $C$ with $0<\mu<\min \left\{\left(q \eta / C_{q}\left(k^{\prime}\right)^{q}\right)^{1 /(q-1)}, 1\right\}$, and $\phi$ be a MKC on $C$ with $0<\gamma<\left(q \mu \eta-C_{q} \mu^{q}\left(k^{\prime}\right)^{q}\right) / q=\tau$. Let $\left\{\alpha_{n}\right\},\left\{\beta_{n}\right\}$ be sequences in $(0,1)$ satisfying the following conditions:

(B1) $\lim _{n \rightarrow \infty} \alpha_{n}=0$;

(B2) $\lim _{n \rightarrow \infty}\left(h_{n} / \alpha_{n}\right)=0$;

(B3) $\sum_{n=1}^{\infty} \alpha_{n}=\infty$;

(B4) $0<\lim \inf _{n \rightarrow \infty} \beta_{n} \leq \lim \sup _{n \rightarrow \infty} \beta_{n}<1$.

Then, $\left\{x_{n}\right\}$ defined by (1.9) converges strongly to a fixed point say $p$ in $F^{*}$ which solves the variational inequality (3.2).

Proof. Since $\alpha_{n} \rightarrow 0$ and $h_{n} / \alpha_{n} \rightarrow 0$ as $n \rightarrow \infty,\left(1-\alpha_{n} \tau\right)\left(h_{n} / \alpha_{n}\right) \rightarrow 0$ as $n \rightarrow \infty$. Thus, $\exists N_{0} \in N$ such that $\left(1-\alpha_{n} \tau\right)\left(h_{n} / \alpha_{n}\right)<(1 / 2)(\tau-\gamma)$ and $\alpha_{n}<\left(k^{\prime}\right)^{-1}$, for all $n \geq N_{0}$. For any point $p \in F^{*}$ and $n \geq N_{0}$,

$$
\begin{aligned}
\left\|y_{n}-p\right\| & =\left\|\alpha_{n}\left(\gamma \phi\left(x_{n}\right)-\mu F p\right)+\left(I-\alpha_{n} \mu F\right) T^{n} x_{n}-\left(I-\alpha_{n} \mu F\right) p\right\| \\
& \leq \alpha_{n} \gamma\left\|x_{n}-p\right\|+\alpha_{n}\|\gamma \phi(p)-\mu F p\|+\left(1-\alpha_{n} \tau\right)\left(1+h_{n}\right)\left\|x_{n}-p\right\| \\
& =\left[1-\alpha_{n}(\tau-\gamma)+\left(1-\alpha_{n} \tau\right) h_{n}\right]\left\|x_{n}-p\right\|+\alpha_{n}\|\gamma \phi(p)-\mu F p\| .
\end{aligned}
$$

But

$$
\left\|x_{n+1}-p\right\| \leq \beta_{n}\left\|x_{n}-p\right\|+\left(1-\beta_{n}\right)\left\|y_{n}-p\right\|
$$

Therefore,

$$
\begin{aligned}
\left\|x_{n+1}-p\right\| & \leq\left[\beta_{n}+\left(1-\beta_{n}\right)\left[1-\alpha_{n}(\tau-\gamma)+\left(1-\alpha_{n} \tau\right) h_{n}\right]\right]\left\|x_{n}-p\right\|+\alpha_{n}\left(1-\beta_{n}\right)\|\gamma \phi(p)-\mu F p\| \\
& =\left[1-\alpha_{n}\left(1-\beta_{n}\right)\left((\tau-\gamma)-\left(1-\alpha_{n} \tau\right) \frac{h_{n}}{\alpha_{n}}\right)\right]\left\|x_{n}-p\right\|+\alpha_{n}\left(1-\beta_{n}\right)\|\gamma \phi(p)-\mu F p\| \\
& \leq\left[1-\alpha_{n}\left(1-\beta_{n}\right)\left[\frac{1}{2}(\tau-\gamma)\right]\right]\left\|x_{n}-p\right\|+\frac{\left(\alpha_{n}\left(1-\beta_{n}\right) / 2\right)(\tau-\gamma)}{(1 / 2)(\tau-\gamma)}\|\gamma \phi(p)-\mu F p\| \\
& \leq \max \left\{\left\|x_{n}-p\right\|, \frac{2\|\gamma \phi(p)-\mu F p\|}{\tau-\gamma}\right\} .
\end{aligned}
$$

By induction, we have

$$
\left\|x_{n}-p\right\| \leq \max \left\{\left\|x_{N_{0}}-p\right\|, \frac{2\|\gamma \phi(p)-\mu F p\|}{\tau-\gamma}\right\}, \quad n \geq N_{0} .
$$


Next we show that

$$
\lim _{n \rightarrow \infty}\left\|x_{n+1}-x_{n}\right\|=0
$$

From (1.9),

$$
\begin{aligned}
y_{n+1}-y_{n}= & \alpha_{n+1} \gamma \phi\left(x_{n+1}\right)+\left(I-\alpha_{n+1} \mu F\right) T^{n+1} x_{n+1} \\
& -\alpha_{n} \gamma \phi\left(x_{n}\right)+\left(I-\alpha_{n} \mu F\right) T^{n} x_{n} .
\end{aligned}
$$

Therefore,

$$
\begin{aligned}
\left\|y_{n+1}-y_{n}\right\|=\| & \alpha_{n+1} \gamma\left(\phi\left(x_{n+1}\right)-\phi\left(x_{n}\right)\right)+\left(\alpha_{n+1}-\alpha_{n}\right) \gamma \phi\left(x_{n}\right) \\
& +\left(I-\alpha_{n+1} \mu F\right) T^{n+1} x_{n+1}-\left(I-\alpha_{n+1} \mu F\right) T^{n+1} x_{n} \\
& +\left(I-\alpha_{n+1} \mu F\right) T^{n+1} x_{n}-\left(I-\alpha_{n} \mu F\right) T^{n+1} x_{n} \\
& +\left(I-\alpha_{n} \mu F\right) T^{n+1} x_{n}-\left(I-\alpha_{n} \mu F\right) T^{n} x_{n} \| .
\end{aligned}
$$

Hence,

$$
\begin{aligned}
\left\|y_{n+1}-y_{n}\right\| \leq & \alpha_{n+1} \gamma\left\|x_{n+1}-x_{n}\right\|+\left|\alpha_{n+1}-\alpha_{n}\right| \gamma\left\|\phi\left(x_{n}\right)\right\| \\
+ & \left(1-\alpha_{n+1} \tau\right)\left(1+h_{n+1}\right)\left\|x_{n+1}-x_{n}\right\|+\left|\alpha_{n+1}-\alpha_{n}\right|\|\mu F\|\left\|T^{n+1} x_{n}\right\| \\
+ & \left(1-\alpha_{n} \tau\right)\left\|T^{n+1} x_{n}-T^{n} x_{n}\right\|, \\
& \quad \limsup _{n \rightarrow \infty}\left(\left\|y_{n+1}-y_{n}\right\|-\left\|x_{n+1}-x_{n}\right\|\right) \leq 0,
\end{aligned}
$$

and by Lemma 2.3

$$
\lim _{n \rightarrow \infty}\left\|y_{n}-x_{n}\right\|=0
$$

Thus, from (1.9),

$$
\left\|x_{n+1}-x_{n}\right\|=\left(1-\beta_{n}\right)\left\|y_{n}-x_{n}\right\| \longrightarrow 0 \text { as } n \longrightarrow \infty
$$

Next, we show that

$$
\lim _{n \rightarrow \infty}\left\|x_{n}-T x_{n}\right\|=0
$$


Since

$$
\begin{aligned}
\left\|x_{n}-T^{n} x_{n}\right\| & \leq\left\|x_{n+1}-x_{n}\right\|+\left\|x_{n+1}-T^{n} x_{n}\right\| \\
& =\left\|x_{n+1}-x_{n}\right\|+\left\|\beta_{n} x_{n}+\left(1-\beta_{n}\right) y_{n}-T^{n} x_{n}\right\| \\
& \leq\left\|x_{n+1}-x_{n}\right\|+\beta_{n}\left\|x_{n}-T^{n} x_{n}\right\|+\left(1-\beta_{n}\right) \alpha_{n}\left(\left\|\gamma \phi\left(x_{n}\right)\right\|+\left\|\mu F T^{n} x_{n}\right\|\right) .
\end{aligned}
$$

Thus,

$$
\left\|x_{n}-T^{n} x_{n}\right\| \leq \frac{1}{1-\beta_{n}}\left\|x_{n+1}-x_{n}\right\|+\alpha_{n}\left(\left\|\gamma \phi\left(x_{n}\right)\right\|+\left\|\mu F T^{n} x_{n}\right\|\right)
$$

Hence,

$$
\lim _{n \rightarrow \infty}\left\|x_{n}-T^{n} x_{n}\right\|=0
$$

Since $T$ is Lipschitz with constant $L$ and for any positive number $n \geq 1$, we have

$$
\begin{aligned}
\left\|x_{n}-T x_{n}\right\| & \leq\left\|x_{n}-T^{n} x_{n}\right\|+\left\|T^{n} x_{n}-T^{n+1} x_{n}\right\|+\left\|T^{n+1} x_{n}-T x_{n}\right\| \\
& \leq\left\|x_{n}-T^{n} x_{n}\right\|+\left\|T^{n} x_{n}-T^{n+1} x_{n}\right\|+L\left\|T^{n} x_{n}-x_{n}\right\| \longrightarrow 0 .
\end{aligned}
$$

Therefore,

$$
\lim _{n \rightarrow \infty}\left\|x_{n}-T x_{n}\right\|=0
$$

Next we show that

$$
\limsup _{n \rightarrow \infty}\left\langle\gamma \phi(p)-\mu F p, J_{q}\left(x_{n}-p\right)\right\rangle \leq 0,
$$

where $p \in F^{*}$ is the unique solution of inequality (3.2). Let $\left\{x_{n_{j}}\right\}$ be a subsequence of $\left\{x_{n}\right\}$ such that

$$
\limsup _{n \rightarrow \infty}\left\langle\gamma \phi(p)-\mu F p, J_{q}\left(x_{n}-p\right)\right\rangle=\lim _{j \rightarrow \infty}\left\langle\gamma \phi(p)-\mu F p, J_{q}\left(x_{n_{j}}-p\right)\right\rangle .
$$

Since $\left\{x_{n}\right\}$ is bounded, we may also assume that there exists some $z \in C$ such that $x_{n_{j}} \rightarrow z$. From (4.11) it follows that

$$
x_{n_{j}}-T x_{n_{j}} \longrightarrow 0 \text { as } j \longrightarrow \infty \text {. }
$$


By Lemma 2.5, the weak limit $z \in C$ of $\left\{x_{n_{j}}\right\}$ is a fixed point of the mapping $T$, so this implies that $z \in F^{*}$. Hence by Theorem 3.1 and $J_{q}$ is a weakly sequentially continuous duality mapping, we have

$$
\limsup _{n \rightarrow \infty}\left\langle\gamma \phi(p)-\mu F p, J_{q}\left(x_{n}-p\right)\right\rangle=\left\langle\gamma \phi(p)-\mu F p, J_{q}(z-p)\right\rangle \leq 0
$$

Finally, we show that $\left\|x_{n}-p\right\| \rightarrow 0$. By contradiction, there is a number $\varepsilon_{0}$ such that

$$
\limsup _{n \rightarrow \infty}\left\|x_{n}-p\right\| \geq \varepsilon_{0}
$$

Case 1. Fixed $\varepsilon_{1}\left(\varepsilon_{1}<\varepsilon_{0}\right)$, if for some $n \geq N \in \mathbb{N}$ such that $\left\|x_{n}-p\right\| \geq \varepsilon_{0}-\varepsilon_{1}$, and for the other $n \geq N \in \mathbb{N}$ such that $\left\|x_{n}-p\right\|<\varepsilon_{0}-\varepsilon_{1}$.

Let

$$
M_{n}=\frac{2 q\left\langle\gamma \phi p-\mu F p, J_{q}\left(y_{n}-p\right)\right\rangle}{\left(\varepsilon_{0}-\varepsilon_{1}\right)^{q}}
$$

From (4.20), we know $\limsup _{n \rightarrow \infty} M_{n} \leq 0$. Hence, there is a number $N$, when $n>N$, we have $M_{n} \leq \tau-\gamma$. We extract a number $n_{0} \geq N$ satisfying $\left\|x_{n_{0}}-p\right\|<\varepsilon_{0}-\varepsilon_{1}$, then we estimate $\left\|x_{n_{0}+1}-p\right\|$

$$
\begin{aligned}
\left\|y_{n_{0}}-p\right\|^{q}= & \left\|\alpha_{n_{0}} \gamma \phi\left(x_{n_{0}}\right)+\left(I-\mu \alpha_{n_{0}} F\right) T^{n_{0}} x_{n_{0}}-p\right\|^{q} \\
= & \left\langle\left(I-\mu \alpha_{n_{0}} F\right) T^{n_{0}} x_{n_{0}}-\left(I-\mu \alpha_{n_{0}} F\right) p, J_{q}\left(y_{n_{0}}-p\right)\right\rangle+\alpha_{n_{0}}\left\langle\gamma \phi\left(x_{n_{0}}\right)-\gamma \phi(p), J_{q}\left(y_{n_{0}}-p\right)\right\rangle \\
& +\alpha_{n_{0}}\left\langle\gamma \phi(p)-\mu F p, J_{q}\left(y_{n_{0}}-p\right)\right\rangle \\
\leq & \left(1-\alpha_{n_{0}} \tau\right)\left(1+h_{n_{0}}\right)\left\|x_{n_{0}}-p\right\|\left\|y_{n_{0}}-p\right\|^{q-1}+\alpha_{n_{0}} \gamma\left\|\phi\left(x_{n_{0}}\right)-\phi(p)\right\|\left\|y_{n_{0}}-p\right\|^{q-1} \\
& +\alpha_{n_{0}}\left\langle\gamma \phi(p)-\mu F p, J_{q}\left(y_{n_{0}}-p\right)\right\rangle \\
< & {\left[1-\alpha_{n_{0}}(\tau-\gamma)+\left(1-\alpha_{n_{0}} \tau\right) h_{n_{0}}\right]\left(\varepsilon_{0}-\varepsilon_{1}\right)\left\|y_{n_{0}}-p\right\|^{q-1}+\alpha_{n_{0}}\left\langle\gamma \phi(p)-\mu F p, J_{q}\left(y_{n_{0}}-p\right)\right\rangle } \\
= & {\left[1-\alpha_{n_{0}}\left[(\tau-\gamma)-\left(1-\alpha_{n_{0}} \tau\right) \frac{h_{n_{0}}}{\alpha_{n_{0}}}\right]\right]\left(\varepsilon_{0}-\varepsilon_{1}\right)\left\|y_{n_{0}}-p\right\|^{q-1} }
\end{aligned}
$$


Journal of Applied Mathematics

$$
\begin{aligned}
& +\alpha_{n_{0}}\left\langle\gamma \phi(p)-\mu F p, J_{q}\left(y_{n_{0}}-p\right)\right\rangle \\
\leq & {\left[1-\frac{\alpha_{n_{0}}}{2}(\tau-\gamma)\right]\left(\varepsilon_{0}-\varepsilon_{1}\right)\left\|y_{n_{0}}-p\right\|^{q-1}+\alpha_{n_{0}}\left\langle\gamma \phi(p)-\mu F p, J_{q}\left(y_{n_{0}}-p\right)\right\rangle } \\
\leq & {\left[1-\frac{\alpha_{n_{0}}}{2}(\tau-\gamma)\right] \frac{1}{q}\left(\varepsilon_{0}-\varepsilon_{1}\right)^{q}+\frac{q-1}{q}\left\|y_{n_{0}}-p\right\|^{q} } \\
& +\alpha_{n_{0}}\left\langle\gamma \phi(p)-\mu F p, J_{q}\left(y_{n_{0}}-p\right)\right\rangle \\
\leq & {\left[1-\frac{\alpha_{n_{0}}}{2}(\tau-\gamma)\right]\left(\varepsilon_{0}-\varepsilon_{1}\right)^{q}+q \alpha_{n_{0}}\left\langle\gamma \phi(p)-\mu F p, J_{q}\left(y_{n_{0}}-p\right)\right\rangle . }
\end{aligned}
$$

But

$$
\begin{aligned}
\left\|x_{n_{0}+1}-p\right\|^{q} & \leq \beta_{n_{0}}\left\|x_{n_{0}}-p\right\|^{q}+\left(1-\beta_{n_{0}}\right)\left\|y_{n_{0}}-p\right\|^{q} \\
& <\beta_{n_{0}}\left(\varepsilon_{0}-\varepsilon_{1}\right)^{q}+\left(1-\beta_{n_{0}}\right)\left\|y_{n_{0}}-p\right\|^{q} .
\end{aligned}
$$

Therefore,

$$
\begin{aligned}
\left\|x_{n_{0}+1}-p\right\|^{q}< & \beta_{n_{0}}\left(\varepsilon_{0}-\varepsilon_{1}\right)^{q}+\left(1-\beta_{n_{0}}\right)\left[1-\frac{\alpha_{n_{0}}}{2}(\tau-\gamma)\right]\left(\varepsilon_{0}-\varepsilon_{1}\right)^{q} \\
& +q \alpha_{n_{0}}\left(1-\beta_{n_{0}}\right)\left\langle\gamma \phi(p)-\mu F p, J_{q}\left(y_{n_{0}}-p\right)\right\rangle \\
= & {\left[1-\frac{1}{2} \alpha_{n_{0}}\left(1-\beta_{n_{0}}\right)(\tau-\gamma)\right]\left(\varepsilon_{0}-\varepsilon_{1}\right)^{q}+q \alpha_{n_{0}}\left(1-\beta_{n_{0}}\right)\left\langle\gamma \phi(p)-\mu F p, J_{q}\left(y_{n_{0}}-p\right)\right\rangle } \\
= & {\left[1-\frac{1}{2} \alpha_{n_{0}}\left(1-\beta_{n_{0}}\right)\left((\tau-\gamma)-M_{n}\right)\right]\left(\varepsilon_{0}-\varepsilon_{1}\right)^{q} } \\
< & \left(\varepsilon_{0}-\varepsilon_{1}\right)^{q} .
\end{aligned}
$$

Hence, we have

$$
\left\|x_{n_{0}+1}-p\right\|<\varepsilon_{0}-\varepsilon_{1} \text {. }
$$

In the same way, we can get

$$
\left\|x_{n}-p\right\|<\varepsilon_{0}-\varepsilon_{1}, \quad \forall n \geq n_{0} .
$$

It contradicts the $\lim \sup _{n \rightarrow \infty}\left\|x_{n}-p\right\| \geq \varepsilon_{0}$.

Case 2. Fixed $\varepsilon_{1}\left(\varepsilon_{1}<\varepsilon_{0}\right)$, if $\left\|x_{n}-p\right\| \geq \varepsilon_{0}-\varepsilon_{1}$, for all $n \geq N \in \mathbb{N}$, from Lemma 2.2, there is a number $r(0<r<1)$ such that

$$
\left\|\phi\left(x_{n}\right)-\phi(p)\right\| \leq r\left\|x_{n}-p\right\|, \quad n \geq N .
$$


It follows (1.9) that

$$
\begin{aligned}
\left\|y_{n}-p\right\|^{q}= & \left\|\alpha_{n} \gamma \phi\left(x_{n}\right)+\left(I-\mu \alpha_{n} F\right) T^{n} x_{n}-p\right\|^{q} \\
= & \left\langle\left(I-\mu \alpha_{n} F\right) T^{n} x_{n}-\left(I-\mu \alpha_{n} F\right) p, J_{q}\left(y_{n}-p\right)\right\rangle+\alpha_{n}\left\langle\gamma \phi\left(x_{n}\right)-\gamma \phi(p), J_{q}\left(y_{n}-p\right)\right\rangle \\
& +\alpha_{n}\left\langle\gamma \phi(p)-\mu F p, J_{q}\left(y_{n}-p\right)\right\rangle \\
\leq & \left(1-\alpha_{n} \tau\right)\left(1+h_{n}\right)\left\|x_{n}-p\right\|\left\|y_{n}-p\right\|^{q-1}+\alpha_{n} \gamma\left\|\phi\left(x_{n}\right)-\phi(p)\right\|\left\|y_{n}-p\right\|^{q-1} \\
& +\alpha_{n}\left\langle\gamma \phi(p)-\mu F p, J_{q}\left(y_{n}-p\right)\right\rangle \\
< & {\left[1-\alpha_{n}(\tau-\gamma r)+\left(1-\alpha_{n} \tau\right) h_{n}\right]\left\|x_{n}-p\right\|\left\|y_{n}-p\right\|^{q-1}+\alpha_{n}\left\langle\gamma \phi(p)-\mu F p, J_{q}\left(y_{n}-p\right)\right\rangle } \\
= & {\left[1-\alpha_{n}\left[(\tau-\gamma r)-\left(1-\alpha_{n} \tau\right) \frac{h_{n}}{\alpha_{n}}\right]\right]\left\|x_{n}-p\right\|\left\|y_{n}-p\right\|^{q-1} } \\
& +\alpha_{n}\left\langle\gamma \phi(p)-\mu F p, J_{q}\left(y_{n}-p\right)\right\rangle \\
\leq & {\left[1-\frac{\alpha_{n}}{2}(\tau-\gamma r)\right]\left\|x_{n}-p\right\|\left\|y_{n}-p\right\|^{q-1}+\alpha_{n}\left\langle\gamma \phi(p)-\mu F p, J_{q}\left(y_{n}-p\right)\right\rangle } \\
\leq & {\left[1-\frac{\alpha_{n}}{2}(\tau-\gamma r)\right] \frac{1}{q}\left\|x_{n}-p\right\|^{q}+\frac{q-1}{q}\left\|y_{n}-p\right\|^{q} } \\
& +\alpha_{n}\left\langle\gamma \phi(p)-\mu F p, J_{q}\left(y_{n}-p\right)\right\rangle \\
\leq & {\left[1-\frac{\alpha_{n}}{2}(\tau-\gamma r)\right]\left\|x_{n}-p\right\|^{q}+q \alpha_{n}\left\langle\gamma \phi(p)-\mu F p, J_{q}\left(y_{n}-p\right)\right\rangle . }
\end{aligned}
$$

Therefore,

$$
\begin{aligned}
\left\|x_{n+1}-p\right\|^{q} \leq & \beta_{n}\left\|x_{n}-p\right\|^{q}+\left(1-\beta_{n}\right)\left\|y_{n}-p\right\|^{q} \\
< & \beta_{n}\left\|x_{n}-p\right\|^{q}+\left(1-\beta_{n}\right)\left[1-\frac{\alpha_{n}}{2}(\tau-\gamma r)\right]\left\|x_{n}-p\right\|^{q} \\
& +\left(1-\beta_{n}\right) \alpha_{n} q\left\langle\gamma \phi(p)-\mu F p, J_{q}\left(y_{n}-p\right)\right\rangle \\
= & {\left[1-\frac{1}{2} \alpha_{n}\left(1-\beta_{n}\right)(\tau-\gamma r)\right]\left\|x_{n}-p\right\|^{q}+\left(1-\beta_{n}\right) \alpha_{n} q\left\langle\gamma \phi(p)-\mu F p, J_{q}\left(y_{n}-p\right)\right\rangle . }
\end{aligned}
$$

By Lemma 2.4, we have that $\left\|x_{n}-p\right\| \rightarrow 0$ as $n \rightarrow \infty$. It contradicts the $\left\|x_{n}-p\right\| \geq \varepsilon_{0}-\varepsilon_{1}$. This completes the proof.

The following corollary follows from Theorem 4.1.

Corollary 4.2. Let $E$ be a q-uniformly smooth and strictly convex Banach space, and let $C$ be a nonempty closed convex subset of $E$ such that $C \pm C \subset C$ and have a weakly sequentially continuous 
duality mapping $J_{q}$ from $E$ to $E^{*}$. Let $T, F^{*}, F, \phi$ and $\left\{\alpha_{n}\right\}$ be as in Corollary 3.2. Let $\left\{x_{n}\right\}$ be defined by

$$
\begin{gathered}
x_{n+1}=\beta_{n} x_{n}+\left(1-\beta_{n}\right) y_{n}, \\
y_{n}=\left(I-\mu \alpha_{n} F\right) T x_{n}+\alpha_{n} \gamma \phi\left(x_{n}\right), \quad \forall n \geq 0,
\end{gathered}
$$

Then, $\left\{x_{n}\right\}$ converges strongly to a fixed point say $p$ in $F^{*}$ which solves the variational inequality (3.2).

\section{References}

[1] J. Diestel, Geometry of Banach Spaces: Selected Topics, vol. 485 of Lecture Notes in Mathematics, Springer, Berlin, Germany, 1975.

[2] K. Goebel and W. A. Kirk, "A fixed point theorem for asymptotically nonexpansive mappings," Proceedings of the American Mathematical Society, vol. 35, pp. 171-174, 1972.

[3] S. Banach, "Surles operations dans les ensembles abstraits et leur application aux equations integrales," Fundamenta Mathematicae, vol. 3, pp. 133-181, 1922.

[4] A. Meir and E. Keeler, "A theorem on contraction mappings," Journal of Mathematical Analysis and Applications, vol. 28, pp. 326-329, 1969.

[5] B. Ali and G. C. Ugwunnadi, "Convergence of implicit and explicit schemes for common fixed points for finite families of asymptotically nonexpansive mappings," Nonlinear Analysis, vol. 5, no. 3, pp. 492-501, 2011.

[6] H. K. Xu, "Inequalities in Banach spaces with applications," Nonlinear Analysis A, vol. 16, no. 12, pp. 1127-1138, 1991.

[7] T. Suzuki, "Moudafi's viscosity approximations with Meir-Keeler contractions," Journal of Mathematical Analysis and Applications, vol. 325, no. 1, pp. 342-352, 2007.

[8] T. Suzuki, "Strong convergence of Krasnoselskii and Mann's type sequences for one-parameter nonexpansive semigroups without Bochner integrals," Journal of Mathematical Analysis and Applications, vol. 305, no. 1, pp. 227-239, 2005.

[9] L. S. Liu, "Ishikawa and Mann iterative process with errors for nonlinear strongly accretive mappings in Banach spaces," Journal of Mathematical Analysis and Applications, vol. 194, no. 1, pp. 114-125, 1995.

[10] H.-K. Xu, "Iterative algorithms for nonlinear operators," Journal of the London Mathematical Society, vol. 66, no. 1, pp. 240-256, 2002.

[11] F. Gu, "Convergence of implicit iterative process with errors for a finite family of asymptotically nonexpansive mappings," Acta Mathematica Scientia A, vol. 26, no. 7, pp. 1131-1143, 2006.

[12] D. S. Mitrinović, Analytic Inequalities, Springer, New York, NY, USA, 1970. 


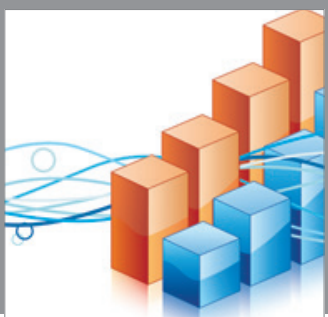

Advances in

Operations Research

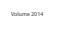

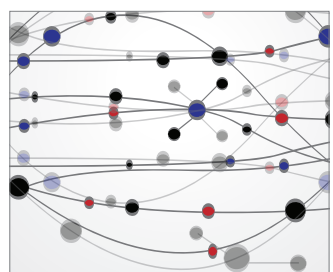

\section{The Scientific} World Journal
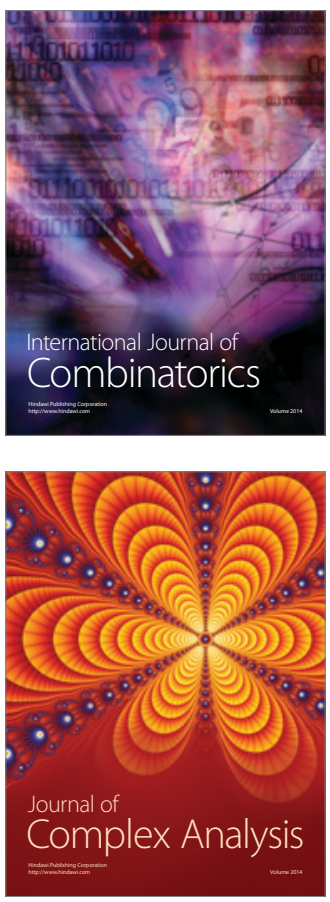

International Journal of

Mathematics and

Mathematical

Sciences
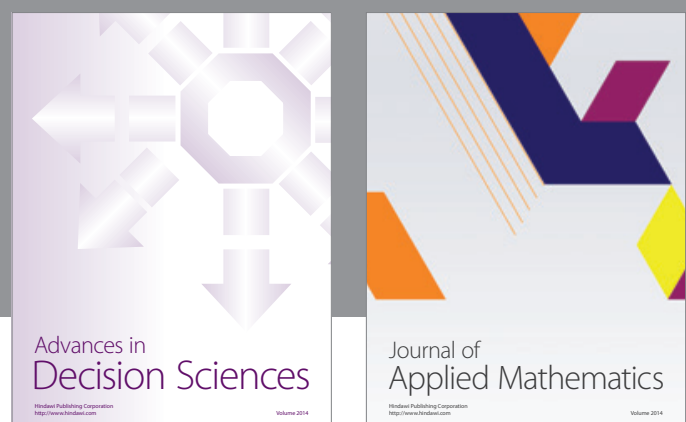

Journal of

Applied Mathematics
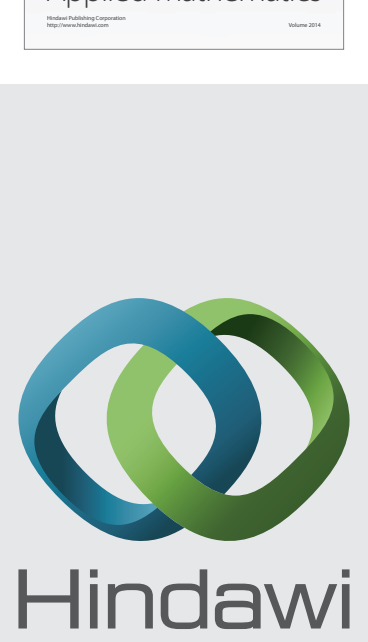

Submit your manuscripts at http://www.hindawi.com
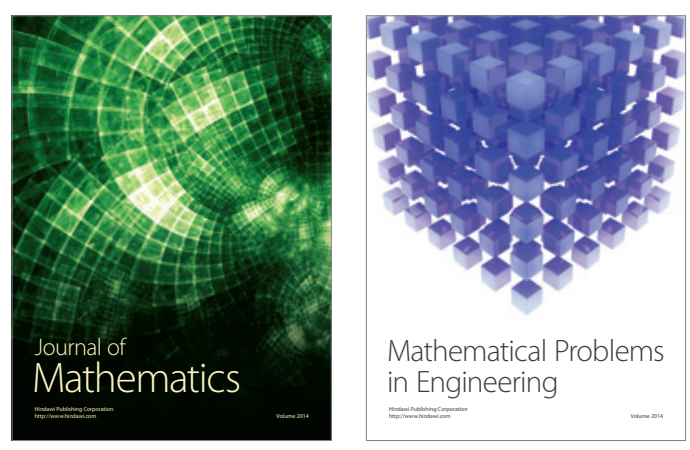

Mathematical Problems in Engineering
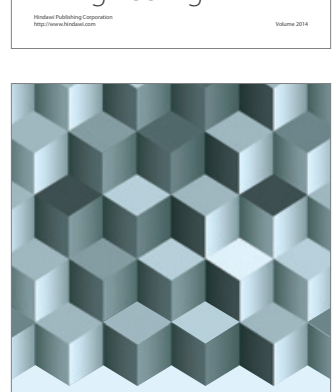

Journal of

Function Spaces
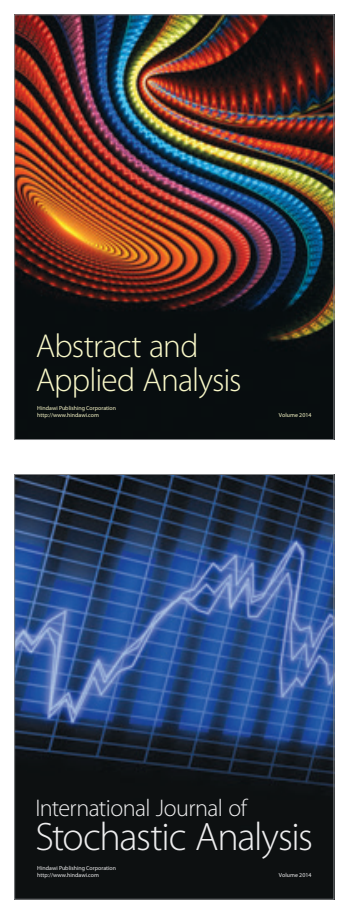

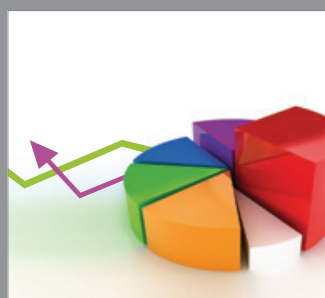

ournal of

Probability and Statistics

Promensencen
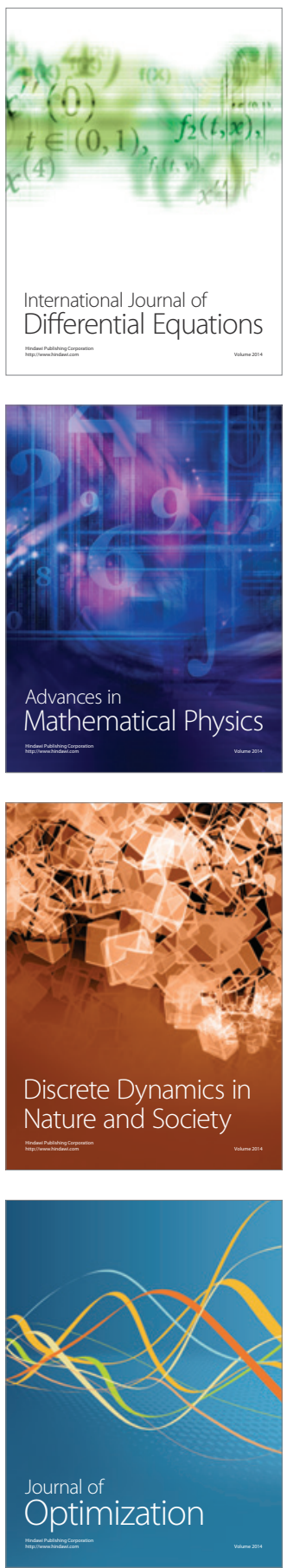